\title{
Historia natural y conservación de los mutualismos planta-animal del bosque templado de Sudamérica austral
}

\author{
Natural history and conservation of plant-animal mutualisms in the temperate forest \\ of southern South America
}

\author{
MARCELO A. AIZEN ${ }^{1}$, DIEGO P. VÁZQUEZ ${ }^{2} \&$ CECILIA SMITH-RAMÍREZ ${ }^{3}$ \\ ${ }^{1}$ Laboratorio Ecotono-CRUB, Universidad Nacional del Comahue, Unidad Postal Universidad, \\ 8400 Bariloche, Río Negro, Argentina; e-mail: marcito@crub.uncoma.edu.ar \\ ${ }^{2}$ Department of Ecology and Evolutionary Biology, University of Tennessee, Knoxville, \\ Tennessee 37996-1610, U.S.A. \\ ${ }^{3}$ Laboratorio de Sistemática y Ecología Vegetal, Facultad de Ciencias, \\ Universidad de Chile, Santiago, Chile
}

\begin{abstract}
RESUMEN
El bosque templado de Sudamérica austral (BTSA) se caracteriza por poseer un biota altamente endémica y una flora que exhibe una de las más altas incidencias de polinización biótica (particularmente ornitofilia) y dispersión animal (particularmente endozoocoria) registradas para cualquier bioma templado. A la luz de importantes avances realizados en los últimos años en el conocimiento de estas interacciones, en este trabajo realizamos una revisión sobre (1) los grupos animales que intervienen en estos mutualismos y su importancia relativa, (2) la estructura comunitaria de estas interacciones, y (3) la existencia de variación geográfica en estas relaciones en base a estudios similares realizados en dos o más localidades a ambos lados de los Andes. También comparamos los mutualismos entre plantas y animales del BTSA con los presentes en otros biomas. Por último, reseñamos la evidencia existente sobre los efectos que la deforestación, la fragmentación, y otras formas de perturbación antrópica están teniendo sobre estos mutualismos. Este trabajo avala la hipótesis de que la alta riqueza de plantas que son polinizadas o dispersadas por vertebrados depende de un ensamble extremadamente pobre de animales mutualistas. Por el contrario, en el caso de la polinización por invertebrados este trabajo revela que las flores entomófilas del BTSA interactúan con un ensamble insospechadamente diverso de insectos, rico en grupos taxonómicos exclusivos de este bioma. Aunque muchos de estos mutualismos parecen ser resilientes, otros aparentan tener un alto grado de sensibilidad a distintas formas de perturbación antrópica.
\end{abstract}

Palabras clave: polinización, dispersión de semillas, invertebrados, vertebrados, Chile, Argentina.

\section{ABSTRACT}

The temperate forest of southern South America (TFSSA) has a highly endemic biota with a flora that exhibits one of the highest incidences of biotic pollination (particularly ornithophily) and animal dispersal (particularly endozoochory) found in any temperate biome worldwide. Much knowledge has been gained in the last few years on the nature of these mutualisms. In this study, we review (1) the group of animals that participate in these relationships and their relative importance, (2) the community structure of these interactions, and (3) their degree of spatial consistency on a geographical scale based on studies conducted in two or more localities at both sides of the Andes. We also compare the plant-animal mutualisms of the TFSSA with those occurring in other biomes. Last, we present evidence showing how deforestation, fragmentation, and other forms of human disturbance are affecting these mutualisms. This work supports the hypothesis that the many plant species pollinated and dispersed by vertebrates in the TFFSA depend on a strikingly poor assemblage of animal mutualists. On the other hand, invertebrate-pollinated plants interact with an unexpectedly diverse assemblage of insect pollinators that include taxa present only in this biome. Although many of these mutualisms seem to be resilient, others appear highly sensitive to different forms of human disturbance.

Key words: pollination, seed dispersal, invertebrates, vertebrates, Chile, Argentina.

\section{INTRODUCCIÓN}

Los mutualismos entre plantas y animales, principalmente los de polinización y de dispersión de semillas, son muy frecuentes en la naturaleza, y son en muchos casos extremadamente importantes para la persistencia espacial y temporal de las especies involucradas (Feinsinger 1987a, Bond 1994). La alteración o completa disrupción de estos mutualismos puede tener efectos en cascada, afectando a otros organismos que no participan directamente en estas relaciones (Nabhan \& Fleming 1993, Kearns \& Inouye 1997, Aizen \& Feinsinger en prensa). Sin embargo, el grado en 
que las plantas dependen de los animales para la polinización y la dispersión de sus semillas varía entre especies y biomas, siendo las floras de los trópicos húmedos aquellas que muestran la mayor dependencia de animales mutualistas y el mayor número de tipos distintos de polinización y dispersión animal (Bawa 1990, Fleming 1991). Las floras templadas, por otro lado, muestran en general una menor incidencia de polinización y dispersión biótica, y una mayor importancia de factores abióticos (e.g., viento) como agentes de transporte de polen y semillas. Una excepción a esta regla la constituyen algunas floras templadas del hemisferio austral que se conformaron bajo el clima más cálido de la antigua Gondwana (Armesto \& Rozzi 1989, Willson et al. 1989, Willson 1991, Aizen \& Ezcurra 1998).

El bosque templado que se extiende como una delgada franja de 100 a $250 \mathrm{~km}$ de ancho a lo largo de la costa pacífica de Sudamérica entre los 37 y $55^{\circ} \mathrm{S}$ (Cabrera \& Willink 1980) representa un ejemplo de este tipo de irregularidad extratropical. Este bioma se caracteriza por poseer una flora que exhibe una de las más altas incidencias de polinización y dispersión animal registradas para cualquier bioma templado (Armesto \& Rozzi 1989, Aizen \& Ezcurra 1998). En el bosque templado de Sudamérica austral, las flores de alrededor del 85 $\%$ de los géneros de plantas leñosas son visitadas y presumiblemente polinizadas por animales. Un rasgo llamativo de este bioma es la polinización por aves (ornitofilia). Cerca del $20 \%$ de los géneros de plantas leñosas producen flores tubulares rojas que son visitadas por la única especie de colibrí, Sephanoides sephaniodes, residente en estos bosques (Ruffini 1992, SmithRamírez 1993, Armesto et al. 1996, Fraga et al. 1997). En cuanto a la dispersión animal, más del $50 \%$ de los géneros de plantas leñosas de este bioma producen frutos carnosos, lo que indica la importancia que tiene la dispersión por vertebrados que ingieren frutos (Armesto et al. 1987, 1996, Aizen \& Ezcurra 1998).

La conservación del bosque templado de Sudamérica austral (BTSA) es considerada una prioridad a nivel mundial (Arroyo \& Hoffman 1997, Dinerstein et al. 1995, Stattersfield et al. 1998). Este bioma contiene una biota rica en endemismos de alta singularidad taxonómica (Heywood 1995). Por ejemplo, el 34 \% de su flora leñosa está representada por géneros endémicos, y existen al menos tres familias de angiospermas (i.e., Misodendraceae, Gomortegaceae y Aextoxicaceae) que sólo se encuentran en el BTSA (Arroyo et al. 1996, Aizen \& Ezcurra 1998). Este alto grado de endemismo se observa también en otros grupos (e.g., anfibios, Duellman 1999; abe- jas, Michener 1979; coleópteros, Morrone \& RoigJuñent 1995) y es probablemente una característica general de esta biota templada. El conocimiento de los principales procesos ecológicos y biogeográficos que determinan el funcionamiento de este ecosistema es importante no sólo desde un punto de vista científico, sino también desde una perspectiva práctica, orientada al desarrollo de pautas que favorezcan la conservación de esta biota única.

Si bien existen revisiones recientes sobre los mutualismos del BTSA (Willson et al. 1996a, Aizen \& Ezcurra 1998), en los últimos cinco años se ha acumulado una gran cantidad de información-mucha de ella todavía inédita o de trabajos en curso-proveniente de localidades ubicadas tanto al oeste (Chile) como al este (Argentina) de la Cordillera de los Andes, donde este bioma alcanza su límite oriental. Un particular avance se ha realizado en el conocimiento de los sistemas de polinización que involucran invertebrados, además del descubrimiento de algunos mutualismos de dispersión muy antiguos que persisten en este bioma, como aquellos donde intervienen reptiles o marsupiales. A la luz de estos nuevos resultados, nuestro principal objetivo en este trabajo es realizar una síntesis sobre (1) los grupos animales que intervienen en estos mutualismos y su importancia relativa, (2) la estructura comunitaria de estas interacciones, y (3) la forma en que estas interacciones varían (o no) geográficamente. También nos proponemos comparar los mutualismos entre plantas y animales del BTSA con los presentes en otros biomas. Por último, dada la potencial importancia que tienen estas interacciones para la conservación de la biota altamente endémica del BTSA, reseñamos la evidencia existente sobre los efectos que la deforestación, la fragmentación, y otras formas de perturbación antrópica están teniendo sobre estos mutualismos.

\section{MUTUALISMOS DE POLINIZACIÓN}

La transferencia de polen de las anteras al estigma de la misma u otra flor (polinización) es un proceso crítico para la reproducción sexual de la mayoría de las angiospermas. Esta transferencia puede ser total o parcialmente mediada por animales, ya sean invertebrados - mayormente insectos- o vertebrados — principalmente aves, pero también mamíferos. En compensación, los animales obtienen una recompensa, mayormente alimento, en forma de néctar o polen. En el caso de plantas polinizadas por animales, la dependencia de mutualistas es total cuando la producción 
de semillas ocurre sólo si el polen es transferido entre individuos sexualmente compatibles. Riveros (1991) estima que un porcentaje muy alto de las especies de la flora del BTSA (ca. $60 \%$ ) requieren obligatoriamente de un animal como vector del polen para su reproducción sexual.

\section{Polinización por invertebrados}

Las interacciones que involucran insectos representan en general uno de los aspectos menos estudiados de la ecología del BTSA, y la polinización no es excepción. Al menos tres estudios, dos de ellos en curso, demuestran que la fauna de invertebrados que visitan flores en el BTSA es más diversa de lo que previamente se había pensado (Aizen \& Ezcurra 1998). Estos tres estudios fueron realizados a una latitud de entre $40-42^{\circ} \mathrm{S}$ por Smith-Ramírez et al. (resultados no publicados) en bosques costeros secundarios de la isla de Chiloé, Chile, en las temporadas de floración de 1999-2000 y 2000-2001; por Riveros et al. (1991) en bosques andinos de Nothofagus dombeyi y Eucryphia cordifolia del Parque Nacional Puyehue, Chile, en 1987-1988; y por Vázquez \& Simberloff (en prensa, resultados no publicados) en bosques andinos de Nothofagus dombeyi del Parque Nacional Nahuel Huapi, Argentina, en 1999-2000 y 2000-2001. En adelante nos referiremos a estos tres estudios a través de las localidades en que fueron realizados.

En general, en los tres sitios estudiados se registró un número relativamente alto de polinizadores, siendo los himenópteros, los dípteros y los coleópteros los grupos más representados. Sin embargo, la proporción del total de las especies de visitantes florales en cada uno de estos grupos varió notablemente entre sitios. En Chiloé se registraron 110 especies de polinizadores visitando las flores de 24 especies de plantas; de estas 110 especies $45(40,9 \%)$ fueron dípteros, 33 (33,6 \%) coleópteros, 16 (14,5\%) himenópteros, tres $(2,7 \%)$ lepidópteros, un $(0,9$ $\%)$ efemeróptero, además de siete especies $(6,4$ $\%$ ) de aves. Por otro lado, en Puyehue se registraron 50 especies de insectos visitando las flores de 31 especies de plantas; de éstas 19 (38\%) fueron dípteros, 19 (38\%) himenópteros, ocho (16\%) coleópteros, y cuatro (8\%) lepidópteros. Por último, en Nahuel Huapi se registraron 131 especies de insectos visitando las flores de 15 especies de plantas del sotobosque de Nothofagus dombeyi. Del total de 97 especies de presumibles polinizadores, $44(45,4 \%)$ fueron himenópteros, $28(28,9 \%)$ dípteros, $21(21,7 \%)$ coleópteros, tres $(3,1 \%)$ lepidópteros, y un $(1 \%)$ heteróptero.
Esta fauna de insectos polinizadores relativamente diversa es, sin embargo, funcionalmente mucho más pobre. Por un lado, una gran cantidad de especies de plantas interactúan con un número relativamente bajo de visitantes florales (Fig. 1). De un modo similar, la mayoría de los insectos polinizadores interactúan con un número relativamente bajo de plantas, y unas pocas especies de insectos son responsables de una gran proporción de las visitas recibidas por las plantas estudiadas (Fig. 2). Por otro lado, aún para aquellas plantas que se relacionan con un número de especies de polinizadores relativamente alto, el número de especies funcionalmente importantes (al menos en términos de la frecuencia de visitas) es probablemente mucho más bajo de lo que podría parecer a priori. Por ejemplo, la especie para la que se registraron la mayor cantidad de potenciales polinizadores en Nahuel Huapi fue el amancay, Alstroemeria aurea (Fig. 1A). Sin embargo, aunque se registraron 53 especies de visitantes florales en esta herbácea, sólo 6 especies fueron responsables del $80 \%$ de las visitas. De un modo similar, Aizen (2001) registró, en tres años consecutivos de observaciones en un bosque de altura de Nothofagus pumilio, que más del $90 \%$ de las visitas al amancay fueron realizadas por su polinizador más eficaz, Bombus dahlbomii (Apidae: Bombini), el único abejorro nativo en el BTSA.

Bombus dahlbomii es a su vez un polinizador extremadamente generalista: tanto en Nahuel Huapi como en Chiloé fue el polinizador que visitó el mayor número de especies de plantas, siendo el principal visitante de las flores de cinco de las 15, y 18 de las 24 especies estudiadas en esas localidades, respectivamente. Lo mismo que otros Bombus, la abundancia de B. dahlbomii se incrementa a lo largo de la temporada de floración (Aizen 2001) de tal forma que su importancia relativa como polinizador es mayor en especies de floración tardía que de floración temprana (Fig. 3).

Otro polinizador muy frecuente en el BTSA es la abeja Cadeguala albopilosa (Colletidae: Diphaglossinae). Esta especie fue el polinizador casi exclusivo del maqui (Aristotelia chilensis) y el principal polinizador de Schinus patagonicus (Anacardiaceae) en Nahuel Huapi, visitando además con menor frecuencia las flores de Berberis darwinii y de la orquídea Gavilea odoratissima. Cadeguala albopilosa es también un polinizador frecuente en Chiloé, siendo responsable del 3,8\% del total de las visitas registradas, visitando siete especies de plantas con flores de tamaños intermedios (i.e., Gaultheria phillyreifolia, Rhaphitamnus spinosus, Hydrangea serratifolia, 
(A) Nahuel Huapi

$\begin{array}{ll}\text { Familia } & \text { Especie } \\ \text { Alstroemeriaceac } & \text { Alstroemeria aurea } \\ \text { Anacardiaceae } & \text { Schinus patagonicus } \\ \text { Rosaceae } & \text { Rosa eglanteria } \\ \text { Asteraceae } & \text { Mutisia decurrens } \\ \text { Berberidaceac } & \text { Berberis darwinii } \\ \text { Asclepiadaceae } & \text { Cynanchum diemii } \\ \text { Eleocarpaceae } & \text { Aristotelia chilensis } \\ \text { Scrophulariacear } & \text { Digitalis purpurea } \\ \text { Saxifragacese } & \text { Ribes magellanicum } \\ \text { Berberidaceae } & \text { Berberis buxifolia } \\ \text { Scrophulariaceae } & \text { Calceolaria crenatiflara } \\ \text { Loranthaceae } & \text { Tristerix corymbosus } \\ \text { Leguminosae } & \text { Vicia nigricans } \\ \text { Orchidaceae } & \text { Gavilea adoratissima } \\ \text { Celasteraceae } & \text { Maytemus chabutensis }\end{array}$

\section{(B) Chiloé}

\begin{tabular}{|c|c|}
\hline Familia & Especie \\
\hline Myrtaceae & Myrceugenia owata \\
\hline Eucrifiaceac & Eucryphia cordifolia \\
\hline Myrtaceae & Amomyrtus meli \\
\hline Myrtaceae & Myrceugenia planipes \\
\hline Cunoniacene & Caldchivia paniculata \\
\hline Proteaceae & Gevuina avellana \\
\hline Myrtaceae & Luma apiculata \\
\hline Myrtaceae & Amomyrtus luma \\
\hline Ericaeae & Gaultheria phyllirelfolia \\
\hline Myrtaceae & Ugni molinase \\
\hline Myrtacese & Myrceugenia parvifolia \\
\hline Verbenaceac & Rhaphithamnus spinosus \\
\hline Hidrangeaceae & Hyfrangra serratifolia \\
\hline Proteaceac & Embothrium coccineum \\
\hline Primulaceae & Anagallis alternifolia \\
\hline Myrtaceae & Ugni candollei \\
\hline Gesneriacene & Asteranthera ovata \\
\hline Ericaeae & Gaultheria mucronata \\
\hline Gesneriaceae & Mitraria coccinea \\
\hline Myrtacease & Myrteola nummularia \\
\hline Philesiaceac & Lururiaga polyphylla \\
\hline Philesiaceac & Luzuriaga radicans \\
\hline
\end{tabular}

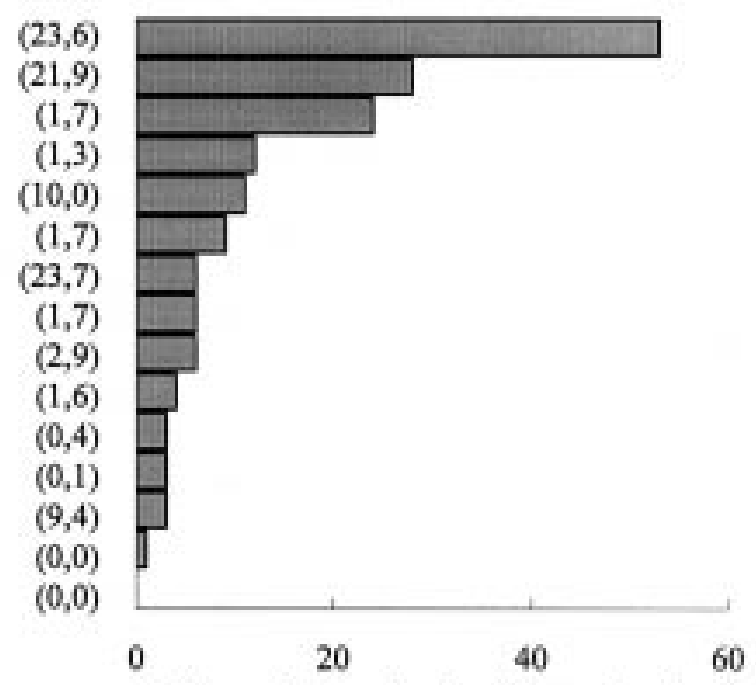

Número de especies de visitantes florales

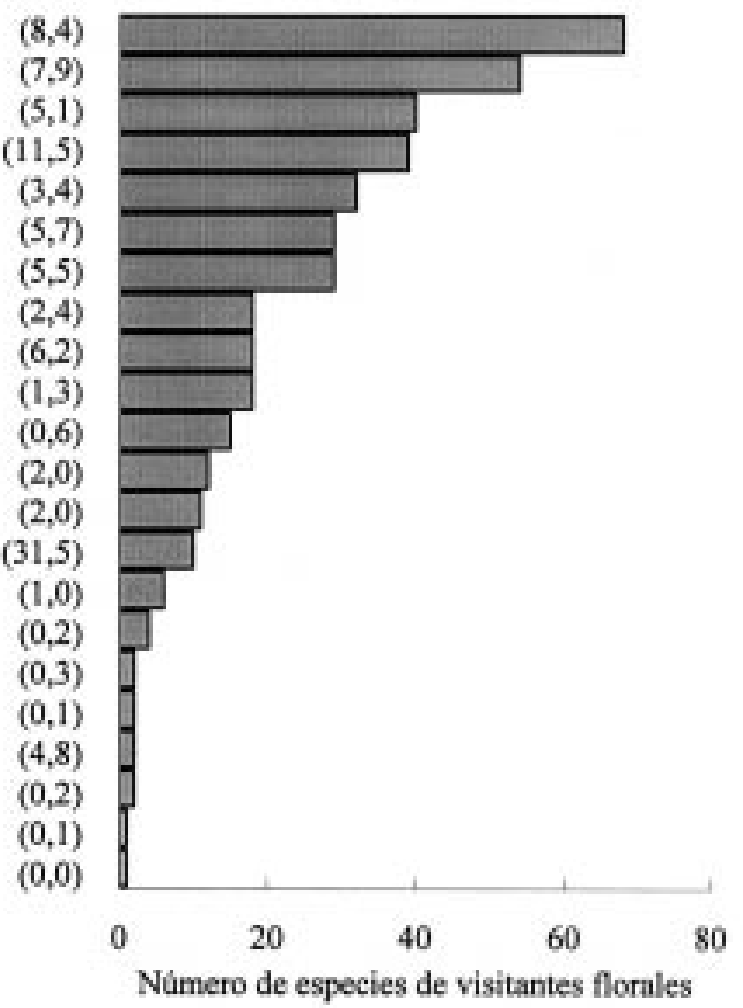

Fig. 1: Número de especies de insectos que visitaron las especies de plantas muestreadas en (A) Nahuel Huapi y (B) Chiloé. Los números entre paréntesis representan el porcentaje total de las visitas de polinizadores recibido por cada una de las especies de plantas. Resultados no publicados de D.P. Vázquez (Nahuel Huapi) y C. Smith-Ramírez (Chiloé).

Number of insect species visiting plant species studied in (A) Nahuel Huapi and (B) Chiloé. Numbers between brackets represent the percentage of total of all pollinator visits received by each plant species. Unpublished results of D.P. Vázquez (Nahuel Huapi) and C. Smith-Ramírez (Chiloé). 


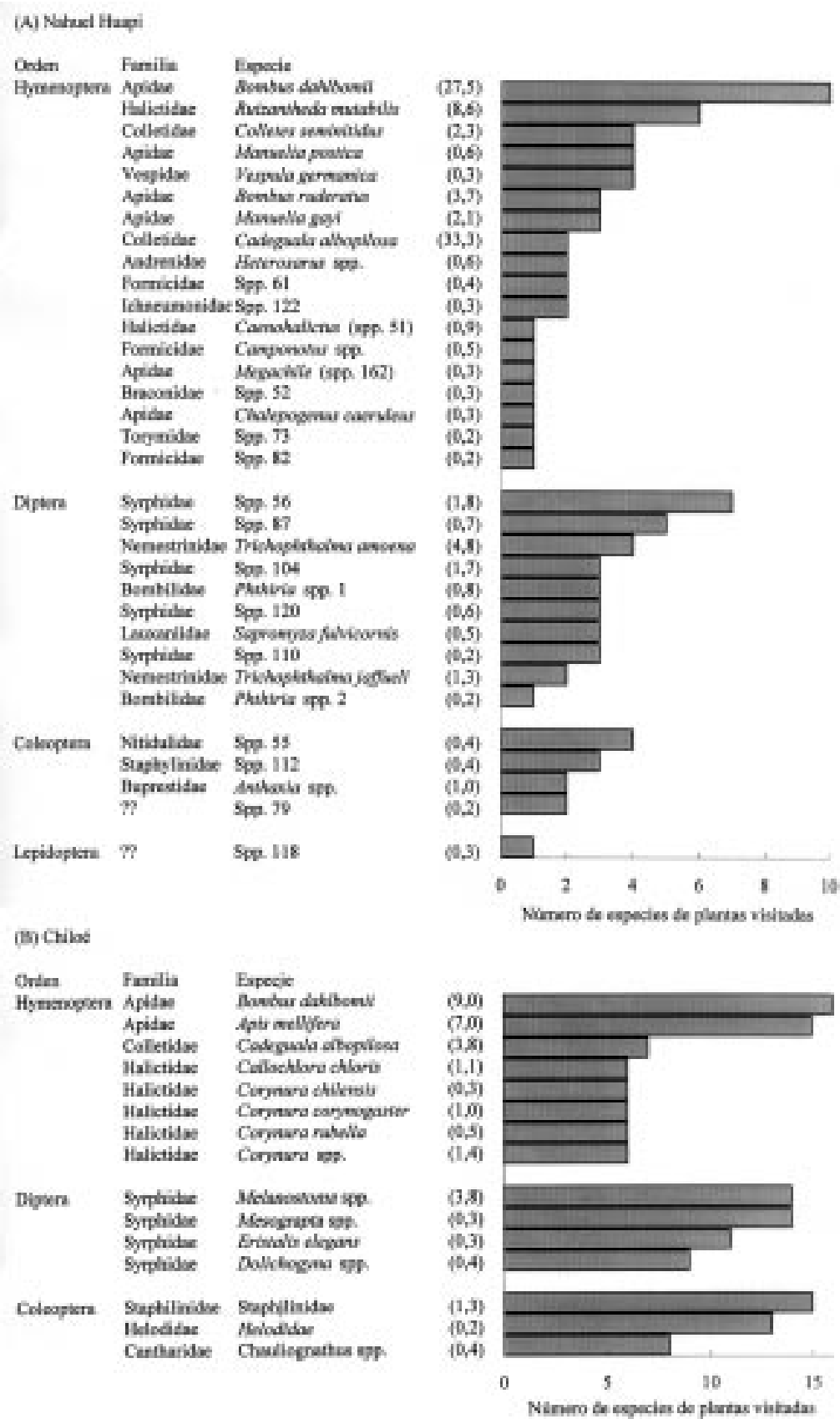

Fig. 2: Número de especies de plantas visitadas (barras) y porcentaje del total de las visitas realizadas (números entre paréntesis) por las especies de polinizadores más abundantes en (A) Nahuel Huapi y (B) Chiloé. En (A) se incluyeron todas las especies de insectos polinizadores que fueron registrados visitando flores al menos 10 veces; las especies de insectos no identificadas se indican con el número correspondiente al catálogo de la colección depositada en el Laboratorio Ecotono, Universidad Nacional del Comahue, Bariloche, Río Negro, Argentina. En (B) se incluyeron sólo las 15 especies más abundantes de insectos polinizadores. Datos no publicados de D.P. Vázquez (Nahuel Huapi) y C. Smith-Ramírez (Chiloé).

Number of plant species visited (bars) and percentage of total visits made (numbers between brackets) by the most abundant pollinator species in (A) Nahuel Huapi and (B) Chiloé. In (A), all insect pollinator species registered visiting flowers at least 10 times were included; unidentified insect species are indicated by a number corresponding to the catalogue of the collection deposited in the Laboratorio Ecotono Universidad Nacional del Comahue, Bariloche, Río Negro, Argentina. In (B), only the 15 most abundant insect pollinator species were included. Unpublished results of D.P. Vázquez (Nahuel Huapi) and C. Smith-Ramírez (Chiloé). 
Ugni sp., Amomyrtus luma, A. meli, y Myrceugenia ovata).

Aunque no tan abundantes como B. dahlbomii y C. albopilosa, muchas otras especies de abejas visitan las flores entomófilas del BTSA. La abeja recolectora de aceite Chalepogenus caeruleus (Apidae) realizó el 73,4 \% de las vistas observadas a las flores de Calceolaria crenatiflora (Scrophulariaceae) en Nahuel Huapi. Esta abeja también visita ocasionalmente otras especies de plantas (Roig-Alsina 1999, Aizen 2001). Otros ápidos relativamente frecuentes en Nahuel Huapi son Manuelia gayi, M. postica, y Svastrides melanura. Además, dos especies de ápidos exóticos son abundantes: el abejorro europeo Bombus ruderatus y la abeja melera Apis mellifera. Bombus ruderatus es relativamente común en Nahuel Huapi (ver sección Efectos de Perturbaciones Antrópicas y Conservación), pero no se lo ha registrado en Chiloé (C Smith-Ramírez resultados no publicados) aunque sí en los alrededores de Valdivia (M.A. Aizen observación personal). Por otro lado, A. mellifera realizó el 6,2\% de las visitas a especies nativas en Chiloé, pero estuvo casi ausente en los bosques de Nothofagus dombeyi de Nahuel Huapi.

Varias especies de abejas halíctidas son relativamente abundantes. Por ejemplo, en Nahuel Huapi Ruizantheda mutabilis realizó el 13,8\% y el 20,7\% de las visitas observadas a Schinus patagonicus y Aristotelia chilensis, respectivamente. Otros halíctidos frecuentes en Nahuel Huapi son varias especies de los géneros Corynura, Caenohalictus, Chilicola, y Evylaeus, mientras que en Chiloé se registraron al menos siete especies en los géneros Callochlora y Corynura, las cuales realizaron en conjunto el 5,6 $\%$ del total de las visitas. También son frecuentes las abejas de la familia Colletidae, entre ellas la ya mencionada Cadeguala albopilosa, además de C. occidentalis, Diphaglossa gayi, y varias especies del género Colletes. Por último, al menos una especie del género Heterosarus (Andrenidae) visita las flores de A. aurea y Mutisia decurrens en Nahuel Huapi.

Otros himenópteros "poco típicos" como polinizadores fueron observados en Nahuel Huapi visitando flores de la enredadera Cynanchum diemii (Asclepiadaceae), en varias oportunidades con polinios adheridos al cuerpo. Estos incluyeron, además de la abeja Colletes seminitidus, parasitoides de las familias Braconidae y Torymidae, y una especie de hormiga.

En el BTSA existe también una rica fauna de dípteros polinizadores, particularmente de la familia gondwánica Nemestrinidae. En Nahuel Huapi, el nemestrínido Trichophthalma amoena fue el visitante floral más frecuente de Berberis buxifolia y Ribes magellanicum, y el segundo más frecuente de Berberis darwinii. Por otro lado, $T$. jaffueli visitó frecuentemente las flores de $A$. aurea. En Chiloé han sido registradas dos especies de nemestrínidos (aún no identificadas) visitando las grandes flores del ulmo, Eucryphia cordifolia. También son relativamente comunes los dípteros de la familia Syrphidae. Al menos 12 especies en esta familia fueron registradas en Chiloé, representando el $17 \%$ de todas las visitas y frecuentando un amplio espectro de tipos de flores, con la excepción de las grandes flores tubulares (Mitraria y Asteranthera) y las flores con forma de campana del género Luzuriaga. En Nahuel Huapi, al menos seis especies de sírfidos son visitantes florales relativamente comunes, siendo responsables del 47,7\% del total de visitas recibidas por la exótica Rosa eglanteria, y del 9,3 $\%$ de las visitas recibidas por Schinus patagonicus. Finalmente, al menos dos especies de bombílidos del género Phthiria, y algunas otras de las familias Acroceridae, Lauxaniidae, Tabanidae, y

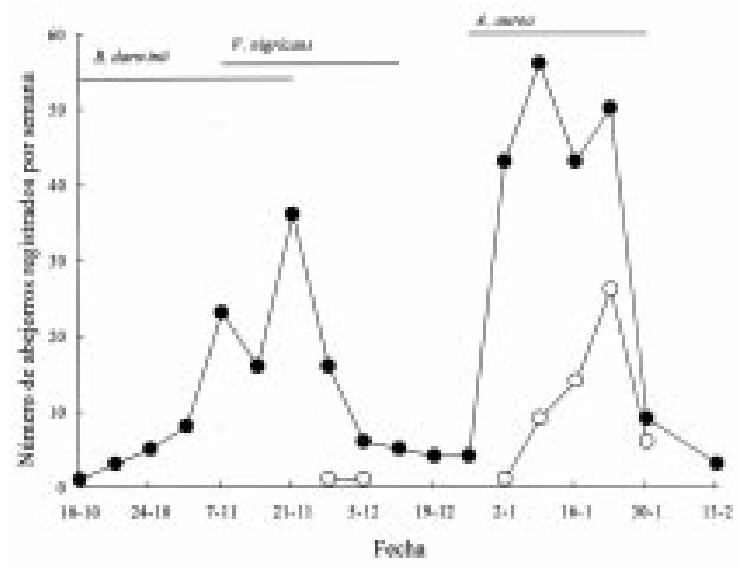

Fig. 3: Número promedio de visitas por semana registradas por D.P. Vázquez (resultados no publicados) para las dos especies de abejorros presentes en los BTSA, en ocho sitios con bosque de Nothofagus dombeyi en la cuenca del Lago Nahuel Huapi, Argentina. Círculos negros: Bombus dahlbomii; círculos blancos: B. ruderatus. Las líneas horizontales en la parte superior del gráfico indican el período de floración de las tres especies más importantes como recursos florales para los abejorros.

Mean number of visits per week recorded by D.P. Vázquez (unpublished results) for the two species of bumblebees present in the TFSSA, in eight sites with Nothofagus dombeyi forest in the basin of the Nahuel Huapi Lake, Argentina. Black circles: Bombus dahlbomii; white circles: $B$. ruderatus. Horizontal lines in the upper part of the graph indicate the flowering period of the three plant species most important as floral resources for the bumblebees. 
Phoridae fueron observadas visitando flores en Nahuel Huapi, aunque es probable que estas especies contribuyan muy poco a la polinización.

Varios coleópteros son también visitantes florales relativamente abundantes. Como hemos mencionado más arriba, en Chiloé se registraron 33 especies de coleópteros visitando flores, aunque sólo realizaron el 5,2 \% del total de las visitas registradas. En Nahuel Huapi, Anthaxia spp. (Buprestidae) realizó el 19,4 \% de las visitas recibidas por Mutisia decurrens y visitó con cierta frecuencia las flores de Alstroemeria aurea. Se registraron además especies de las familias Mordellidae, Nitidulidae, Salpingidae y Staphilinidae.

En general, los lepidópteros representan una proporción pequeña del total de los polinizadores en el BTSA, tanto en el número de especies (ver datos presentados más arriba) como en la frecuencia de visitas: en Chiloé y en Nahuel Huapi, el porcentaje de visitas por lepidópteros diurnos registrado fue inferior al 0,01\%. Sin embargo, en Nahuel Huapi se observaron individuos de varias especies de polillas (aún no identificadas) atrapados en las flores de la asclepiadácea Cynanchum diemii. Estas polillas tienen muy probablemente actividad nocturna, y es posible que intervengan en la polinización de esta especie y de otras como las del género Azara (M.A. Aizen observación personal). En los bosques de Chiloé existen al menos 18 especies de mariposas diurnas y entre 40-50 especies de polillas nocturnas (A. Angulo comunicación personal), por lo que se espera que un estudio más extensivo pueda determinar el papel de estas especies en la polinización.

\section{Polinización por vertebrados}

De todas las aves del BTSA, sin duda el colibrí Sephanoides sephaniodes es el polinizador vertebrado más importante y abundante del BTSA. En verano, en la isla de Chiloé llega a ser la especie de ave más común con el $16,5 \%$ de la abundancia relativa de acuerdo a censos visualesauditivos (Díaz et al. resultados no publicados). En los bosques costeros de Osorno a fines de verano, se constituye en la segunda especie mas frecuente después del chucao (Scelorchilus rubecula) con un $11 \%$ de abundancia relativa;

${ }^{1}$ CHALCOFF V, MA AIZEN \& L GALLETO (2001) La composición del néctar de la flora ornitófila del bosque templado austral y las preferencias del colibrí Sephanoides sephaniodes. I Reunión Binacional de Ecología, San Carlos de Bariloche, Libro resúmenes: 260. mientras que en el bosque de Llao-Llao, en las cercanías de Bariloche, es la cuarta especie estival más común con un $9 \%$ de abundancia relativa (Amico 2000). A pesar de ser una especie que migra hacia latitudes más bajas durante el invierno, una parte de la población permanece todo el año como residente en la zona norte (por encima de $\operatorname{los} 42^{\circ} \mathrm{S}$ ) de los bosques del sur de Argentina y Chile. En esta época se la ha registrado en los bosques preandinos de Osorno contribuyendo con el 10 al $20 \%$ de la abundancia relativa de la avifauna (García 1982), y en Chiloé con el $25 \%$ de la abundancia relativa de aves invernales (Sabag 1993, Díaz et al. resultados no publicados). Aunque su abundancia relativa no ha sido cuantificada, también es muy frecuente en los bosques de la región del Nahuel Huapi durante esta estación (M.A. Aizen observación personal).

Para subsistir en el clima relativamente frío que caracteriza al BTSA, este colibrí requiere consumir grandes cantidades de azúcar. Las flores que visita presentan abundante néctar, el cual oscila entre 0,8 a 23,0 $\mu 1$ en Chiloé (Smith-Ramírez 1993) y 1,9 a $15,9 \mu \mathrm{l}$ en Puerto Blest, en el extremo oeste del lago Nahuel Huapi (Fraga et al. 1997); con una concentración de azúcar que va entre 8,4 a $30,1 \%$ peso/peso (Chiloé) y 23,0 a $38,0 \%$ (Puerto Blest). Como se ha encontrado en floras neotropicales (Baker \& Baker 1983), el néctar de las flores del BTSA visitadas por el colibrí S. sephaniodes es rico en sacarosa $(71,3 \%$ del total de azúcares, promedio de nueve especies) y pobre en monosacáridos (16,6\% fructosa, y $12,1 \%$ glucosa). Estos porcentajes coincidieron con el orden de preferencia por soluciones artificiales de estos tres azúcares que exhibe este colibrí en ensayos de campo (Chalcoff et al. 2001) ${ }^{1}$.

Es de notar que no todas las especies visitadas por S. sephaniodes estudiadas en Chiloé están presentes en Puerto Blest. En general, existe una mayor riqueza de plantas ornitófilas en los bosques costeros y andinos de la vertiente occidental de los Andes que en la vertiente oriental (Tabla 1). El papel de Sephanoides sephaniodes como polinizador de las especies ornitófilas listadas en la Tabla 1 ha sido corroborado por la presencia de polen de la mayoría de ellas en el cuello, cabeza y pico de los picaflores (Ruffini 1992, SmithRamírez 1993, Fraga et al. 1997). Aunque los machos de esta especie defienden frecuentemente territorios florales, el análisis de la composición de las cargas polínicas que llevan los picaflores en sus cuerpos también sugiere que los mismos pueden tener rutas de forrajeo de varios kilómetros de largo, incluyendo localidades ubicadas a 
ambos lados de la cordillera de Los Andes (Ruffini 1992).

Las especies con flores tubulares grandes visitadas por $S$. sephaniodes son también visitadas por el abejorro Bombus dahlbomii, desconociéndose la eficacia relativa de polinización de estos dos animales. Se ha descrito que en los meses de verano Patagonas gigas llega a los bosques valdivianos hasta los $40^{\circ} \mathrm{S}$ (Araya \& Millie 1986), pero se desconoce las especies de plantas que visita. Las flores tubulares pequeñas son visitadas, además de $S$. sephaniodes, por un grupo más diverso de polinizadores, los cuales incluyen himenópteros, mariposas y dípteros (Riveros 1991, Ruffini 1992, C. Smith-Ramírez resultados no publicados).

La floración de la mayoría de las especies ornitófilas en los bosques andinos ocurre principalmente en los meses de noviembre a febrero (Riveros 1991, Fraga et al. 1997), al igual que ocurre en el bosque costero de Chiloé. Sin embargo, a diferencia del bosque andino, en el bosque costero se encuentra un mayor número de especies ornitófilas en floración durante todo el año (Smith-Ramírez \& Armesto 1994). Entre las especies ornitófilas que florecen en los meses de invierno se encuentran Tristerix corymbosus, Lapageria rosea, Latua pubiflora, Campsidium valdivianum y Sophora microphylla; las tres últimas comienzan su floración a fines de julio y permanecen en flor hasta la llegada de la primavera austral. Sin embargo, por su extensa floración-la que incluye todos los meses del invierno-, amplia distribución y abundancia, las flores de $T$. corymbosus resultan particularmente importantes para la permanencia invernal de las poblaciones de $S$. sephaniodes en los bosques andinos y en el bosque de Chiloé (Smith-Ramírez 1993, M. A. Aizen resultados no publicados). Prueba de ello es que mientras en el verano este colibrí se distribuye por los bosques hasta el extremo sur del continente $\left(55^{\circ} \mathrm{S}\right)$, en invierno el límite austral de su distribución coincide con el de esta hemiparásita $\left(42^{\circ} \mathrm{S}\right)$ (Araya \& Millie 1986, Kuijt 1988).

En el BTSA existe una asociación entre la forma de vida y la ornitofilia. Mientras el $37 \%$ de los géneros epifíticos leñosos del BTSA presentan este síndrome floral-siendo todos ellos visitados y presumiblemente polinizados por $S$. sephanioides-, este porcentaje decae a un $17 \%$ en el caso de los arbustos y a un $4 \%$ (una especie) en el caso de los árboles (Aizen \& Ezcurra 1998). A pesar de que algunas especies del género Ourisia presentan claramente el síndrome de la ornitofilia, hasta el presente no hay evidencia de que $S$. sephaniodes visite, al menos con cierta frecuen-

TABLA 1

Presencia (X) y ausencia (-) de especies de plantas ornitófilas en cuatro localidades del bosque templado de Sudamérica austral entre 40 y $42^{\circ} \mathrm{S}$. Las localidades están ordenadas de la más oriental (Llao-Llao) a la más occidental (Chiloé) (datos del Anexo I en Aizen \& Ezcurra 1998)

Presence (X) and absence (-) of ornitophilous plant species in four localities of the temperate forest of southern South America between 40 and $42^{\circ} \mathrm{S}$. Localities are ordered from the eastern- (Llao-Llao) to the western-most (Chiloé) one (data from Appendix I in Aizen \& Ezcurra 1998)

\begin{tabular}{|c|c|c|c|c|}
\hline \multirow[t]{2}{*}{ Especie } & \multicolumn{2}{|c|}{ Argentina } & \multicolumn{2}{|c|}{ Chile } \\
\hline & Llao-Llao & Puerto Blest & Antillanca & Chiloé \\
\hline Asteranthera ovata (Gesneriaceae) & - & $\mathrm{X}$ & $\mathrm{X}$ & $X$ \\
\hline Campsidium valdivianum (Bignoniaceae) & - & $\mathrm{X}$ & $X$ & $X$ \\
\hline Crinodendron hookerianum (Elaeocarpaceae) & - & - & - & $\mathrm{X}$ \\
\hline Desfontainia spinosa (Desfontainiaceae) & $\mathrm{X}$ & $\mathrm{X}$ & $\mathrm{X}$ & $\mathrm{X}$ \\
\hline Embothrium coccineum (Proteaceae) & $X$ & $\mathrm{X}$ & $X$ & $X$ \\
\hline Escallonia rubra (Escaloniaceae) & $\mathrm{X}$ & $\mathrm{X}$ & $\mathrm{X}$ & $\mathrm{X}$ \\
\hline Fascicularia bicolor (Bromeliaceae) & - & - & - & $\mathrm{X}$ \\
\hline Fuchsia magellanica (Onagraceae) & $\mathrm{X}$ & $\mathrm{X}$ & $\mathrm{X}$ & $\mathrm{X}$ \\
\hline Mitraria coccinea (Gesneriaceae) & - & $\mathrm{X}$ & $\mathrm{X}$ & $\mathrm{X}$ \\
\hline Notanthera heterophylla (Lorantaceae) & - & - & - & $\mathrm{X}$ \\
\hline Philesia magellanica (Philesiaceae) & - & - & - & $\mathrm{X}$ \\
\hline Rhaphitamnus spinosus (Verbenaceae) & $X$ & $X$ & $X$ & $X$ \\
\hline Sarmienta repens (Gesneriaceae) & - & - & $\mathrm{X}$ & $\mathrm{X}$ \\
\hline Tristerix corymbosus (Loranthaceae) & $\mathrm{X}$ & - & $\mathrm{X}$ & $\mathrm{X}$ \\
\hline Total & 6 & 8 & 10 & 14 \\
\hline
\end{tabular}


cia, las flores de especies de hierbas nativas (Arroyo \& Peñaloza 1990, Smith-Ramírez 1993, Fraga et al. 1997).

En el BTSA existen al menos dos referencias de nectarivoría por paseriformes. En un estudio realizado en la isla de Chiloé, Smith-Ramírez \& Armesto (1998) encontraron que las flores de la proteácea Embothrium coccineum fueron visitadas por nueve especies de aves-incluyendo el picaflor S. sephaniodes- y el himenóptero Diphaglossa gayi. Una especie, el psittácido Enicognathus leptorynchus, se comportó como "ladrona" de néctar, destruyendo las flores de este árbol. Las otras especies consumieron néctar con baja frecuencia, con la notable excepción del tiránido Elaenia albiceps, el cual aportó el 76,6\% del total de las visitas. Tanto los individuos de $E$. albiceps y $S$. sephaniodes capturados presentaron polen de E. coccineum. Sin embargo, los autores estimaron que E. albiceps transportó en total 27 veces más granos de polen que $S$. sephaniodes. Cabe destacar que la nectarivoría de este paseriforme en flores de E. coccineum sólo se ha registrado en la isla de Chiloé (Smith-Ramírez \& Armesto 1998), y en la cordillera de La Costa y precordillera occidental andina a $\operatorname{los} 41^{\circ} \mathrm{S}$ (E Couve \& C Smith observaciones personales). En un estudio realizado en el Parque Nacional Lanín, Argentina, Montaldo et al. (1999) ${ }^{2}$ registraron depredación de flores de esta proteácea por parte del psittácido Enicognatus ferrugineus y del fringílido Phrygilus gayi. A pesar de numerosas observaciones de visitas a flores de E. coccineum por $S$. sephaniodes, himenópteros y dípteros, no se han registrado visitas por E. albiceps en la vertiente oriental de la cordillera de Los Andes (M.A. Aizen observación personal) ni en la vertiente occidental ca. $40^{\circ} \mathrm{S}$ (Riveros et al. 1991, C. Smith-Ramírez observación personal), a pesar de ser el ave más abundante en los bosques andinos durante la época de la floración de esta especie. Estas diferencias podrían estar relacionadas con la imposibilidad de asimilar sacarosa que tendrían la mayoría de los paseriformes (Martínez del Río et al. 1992). M.A. Aizen \& L. Galetto (resultados no publicados) encontraron que la proporción relativa de sacarosa con respecto al total de azúcares en poblaciones de E. coccineum de la región de Nahuel Huapi, en la vertiente oriental de los Andes, duplica el $40 \%$ encontrado por Smith-Ramírez \& Armesto (1998) en su sitio de estudio en Chiloé.

${ }^{2}$ MONTALDO NH, GG ROITMAN, M DEVOTO \& D MEDAN (1999) Biología reproductiva de Embothrium coccineum (Proteaceae) en la Provincia de Neuquén. XIX Reunión Argentina de Ecología, Tucumán, Libro resúmenes: 106.
El segundo caso de nectarivoría por paseriformes fue registrado por Traveset et al. (1998) en Tierra del Fuego. En este estudio las flores ornitófilas de Fuchsia magellanica fueron visitadas primariamente por el fringílido Phrygilus patagonicus con el $80 \%$ del total de las visitas, y secundariamente por $S$. sephaniodes $(11 \%$ de las visitas) y el tiránido E. albiceps (9\% de las visitas). Sin embargo, $P$. patagonicus no se comportó como polinizador sino como ladrón de néctar, reduciendo la producción de frutos de este arbusto en un $20 \%$. Esta disminución en la reproducción se debió principalmente al daño que causaban a los ovarios cuando agujereaban con sus picos las bases de las corolas en busca de néctar. La nectarivoría por paseriformes en $F$. magellanica no fue observada en la zona norte de los bosques (M.A. Aizen observación personal).

La importancia de la polinización por aves en la flora del BTSA contrasta con la ausencia de polinización por otros vertebrados. Hasta el presente no se conocen casos de polinización por mamíferos o reptiles en este bioma.

\section{MUTUALISMOS DE DISPERSIÓN}

Los vertebrados son los dispersores de semillas más importantes del BTSA. La granivoría y eventual dispersión por hormigas (Hymenoptera: Formicidae) es un fenómeno observado en ecosistemas áridos y semiáridos vecinos como la estepa patagónica y el desierto del Monte (Folgarait et al. 1998, Marone et al. 2000), pero su ocurrencia en el BTSA es desconocida (Willson 1991, pero ver Villagrán et al. 1986). Al menos en la flora de este bioma boscoso no se encuentran semillas con elaiosomas (i.e., apéndice de naturaleza lipídica) que indicarían una especialización a este modo de dispersión. La dispersión de semillas o frutos que se adhieren al pelaje de mamíferos y aves (epizoocoria) es relativamente frecuente, aunque está restringido a especies herbáceas del sotobosque (e.g., Acaena, Osmorhiza, Uncinia, Madia, Myosotis, entre otras). Sin embargo, no podemos considerar un mutualismo a este modo de dispersión ya que la contraparte animal no obtendría un beneficio aparente.

Sin lugar a duda el tipo de dispersión más frecuente en el BTSA y que sí se considera un verdadero mutualismo es la endozoocoria, que involucra la ingestión de frutos carnosos por vertebrados y el regurgitamiento o defecación de las semillas (Armesto et al. 1987, 1996). El transporte de la semillas a micrositios distantes de la planta madre y/o el pasaje de la semillas por el tracto digestivo de sus dispersores en muchos 
casos incrementa la germinación de las semillas y el éxito en el establecimiento (Traveset 1998). La pulpa de los frutos o los arilos carnosos que rodean a las semillas (e.g., Maytenus, Podocarpus, Saxe-gothaea) representan, por otro lado, la recompensa alimenticia que obtienen los dispersores de semillas por su servicio.

Las aves y los mamíferos, en ese orden, representan los principales grupos de dispersores del BTSA. Si bien la dispersión por reptiles sería menos relevante en este bioma (Willson 1991), un estudio reciente realizado en la Isla de Chiloé muestra que la lagartija Liolaemus pictus consume frutos y defeca semillas de dos hierbas rubiáceas del sotobosque, Nertera granadensis y Relbunium hypocarpium, y de un arbusto ericáceo (Gaultheria sp.). En N. granadensis, el pasaje de las semillas por el tracto digestivo de la lagartija incrementa su germinación (Willson et al. 1996b). Las lagartijas podrían también estar involucradas en la dispersión de las especies del género Gunnera (Armesto \& Rozzi 1989). Sin embargo, no existen aún estudios que confirmen esta conjetura.

Aunque entre 15 y 20 especies de aves han sido identificadas como parcialmente frugívoras en el BTSA (Armesto et al. 1987, Grigera et al. 1994, Rozzi et al. 1996), sólo dos paseriformes (el fíofío, Elaenia albiceps, y el zorzal, Turdus falcklandii) serían dispersores importantes tanto por su abundancia como por la frecuencia con que consumen frutos. Existen hasta el momento estudios para dos localidades geográficas contrastantes, una al oeste y otra al este de la Cordillera de Los Andes, que apoyarían la hipótesis de que la dispersión de la mayoría de especies de plantas que producen frutos carnosos en este bioma depende principalmente de estas dos especies. En un estudio realizado por Sabag (1993) en la Isla de Chiloé, Elaenia albiceps representó cerca del $50 \%$ de todas las capturas realizadas con redes de niebla y el $85 \%$ de las capturas de aves frugívoras durante un período de 4 años (1988-1991). Este autor encontró semillas intactas y/o restos de pulpa en las heces de todos los individuos capturados de este tiránido. Las especies de plantas identificadas en las heces, 10 en total (e.g., Drimys winteri, Amomyrtus luma, Myrceugenia ovata, dos especies de Berberis y dos de Gaultheria, entre otras), representaron casi la mitad de las especies con frutos carnosos en el área de estudio; aunque como fue señalado por Armesto et al. (1996) este número es seguramente una subestimación de la diversidad de frutos consumidos por E. albiceps, ya que el análisis de su dieta estuvo restringido a un breve período del mes de enero. Sin embargo, a pesar de su importancia numérica, su papel como dispersor está restringido a los meses de octubre a marzo, período en el que está presente en el BTSA. Por ejemplo, Hernández (1995) encontró que Elaenia albiceps contituyó el $58 \%$ del total de visitas registradas a árboles de Drimys winteri y más del $74 \%$ a árboles de Amomyrtus luma; ambas especies de fructificación estival. Por otro lado, en el estudio de Sabag (1993) Turdus falcklandii fue mucho menos abundante que E. albiceps (5 versus $44 \%$ del total de las capturas), pero su status de frugívoro frecuente y de residente anual lo convierten en el principal dispersor de muchas especies que fructifican entre el otoño y la primavera. Por ejemplo, Willson et al. (1996c) encontraron en Chiloé que $T$. falcklandii es el principal dispersor de las semillas ariladas de Podocarpus nubigena, una conífera que fructifica en la primavera temprana.

Resultados similares a los de Sabag (1993) fueron hallados por Amico (2000) para el bosque de LlaoLlao, en la cuenca del Nahuel Huapi. Durante dos estaciones de muestreo (1998-1999 y 1999-2000), E. albiceps y $T$. falcklandii representaron el 52 y el $9 \%$ del total de las capturas realizadas con redes de niebla, respectivamente. Este autor encontró semillas intactas y/o restos de pulpa en los contenidos estomacales de todos los individuos de E. albiceps y en el $75 \%$ de los de T. falcklandii. En estos contenidos estuvieron presentes siete (E. albiceps) y seis ( $T$. falcklandii) de las nueve especies productoras de frutos carnosos presentes en el sitio de estudio. Mientras que Schinus patagonicus, Aristotelia chilensis, y Azara microphylla fueron las especies más frecuentes en los contenidos estomacales de E. albiceps, las especies A. chilensis, $A$. microphylla, Luma apiculata, y Berberis sp. fueron las más representadas en los de T. falcklandii (Amico 2000). A pesar de las diferencias en la composición y diversidad vegetal que existen a lo largo de los abruptos gradientes ambientales que caracterizan el BTSA (Aizen \& Ezcurra 1998), estos trabajos (Sabag 1993, Amico 2000) demuestran que estas dos aves tendrían un papel central como dispersores de semillas en todo este bioma (ver Armesto et al. 1996, Willson et al. 1996a). Aunque menos importantes, Curaeus curaeus y Phytotoma rara fueron registradas cómo consumidoras de frutos de varios arbustos (Sabag 1993, Hernández 1995). Correa et al. (1990) proponen también que el chucao (Scelorchilus rubecula), una especie típicamente omnívora, podría ser un dispersor efectivo de las semillas de varias especies como las mirtáceas Amomyrtus luma y Myrteola nummularia.

En contraste con los trópicos y subtrópicos, donde existe una gran cantidad de especies de murciélagos frugívoros, las especies de quirópteros presentes en el BTSA serían básicamente insectívoras. Sin embargo, se conoce muy poco 
sobre la ecología de los murciélagos de este bioma (Jaksic 1997) y se necesitarían estudios más detallados sobre la dieta y el comportamiento de estos mamíferos antes de poder descartar su papel como dispersores.

Posiblemente, el mamífero no volador más abundante en el estrato arbustivo y arbóreo del BTSA por encima de los $42^{\circ} \mathrm{S}$ sea el monito del monte, Dromiciops gliroides (Rau et al. 1995, Amico 2000). Este marsupial, considerado un verdadero fósil viviente, es el único representante actual del orden Microbiotheria (Spotorno et al. 1997). Armesto et al. (1987) señalan que si bien el monito del monte consume frutos (ver también Meserve et al. 1988, Kelt \& Martínez 1989) su papel como dispersor de semillas es desconocido. Recientemente Amico \& Aizen (2000) identificaron a este marsupial como único dispersor del quintral, Tristerix corymbosus (Loranthaceae), en el bosque de Llao-Llao. Este es el único caso conocido hasta el presente de dispersión de un muérdago por un mamífero. Los resultados de este trabajo muestran que este animal no sólo consume una gran cantidad de frutos de quintral defecando las semillas intactas, sino que deposita cerca del $90 \%$ de las mismas sobre ramas a las que se adhieren gracias al mucílago que las rodea. Además, el pasaje de la semilla por el tracto digestivo es crucial para el establecimiento del quintral, ya que de otra forma la germinación no ocurre. La alta dependencia que tiene el quintral por su dispersor marsupial podría estar reflejando la antigüedad de esta relación que podría haberse originado en los bosques de Gondwana occidental hace más de 70 millones de años. Esta relación lorantácea-marsupial antecedería en casi 50 millones de años las interacciones más modernas entre distintos muérdagos y sus aves dispersoras (Reid 1991, Tambussi \& Noriega 1996). En el matorral de Chile central, donde D. gliroides está ausente, esta lorantácea es dispersada por tres especies de aves, Turdus falcklandii, Mimus thenca, y Curaeus curaeus (Hoffman et al. 1986).

De los mamíferos terrestres que se encuentran en el BTSA, los zorros (Pseudolapex spp.) serían dispersores de semillas importantes de varias especies, particularmente en paisajes fragmentados. Los datos sobre consumo de frutos por el zorro de Darwin (Pseudalopex fulvipes), demuestran que los mamíferos podrían estar dispersando las semillas de muchas plantas que también son dispersadas por aves (Armesto et al. 1996). El ciervo enano, Pudu pudu, ha sido también citado como parcialmente frugívoro (Grigera et al. 1994, Armesto et al. 1987) pero su importancia como dispersor de semillas en el BTSA todavía no se ha evaluado.

\section{COMPARACIÓN CON OTROS BIOMAS}

La prevalencia de diferentes tipos de mutualismos entre plantas y polinizadores en el BTSA está entre las más altas registradas para ecosistemas templados y es, en muchos aspectos, comparable con la de ecosistemas tropicales. Como mencionamos anteriormente, las aves polinizan el $23 \%$ (14 especies) de las especies de plantas leñosas en Chiloé (Smith-Ramírez 1993, Smith-Ramírez \& Armesto 1994, Armesto et al. 1996), y aunque este porcentaje disminuye considerablemente en sentido oeste-este (de un 24,6\% de los géneros de plantas leñosas en los bosques costeros de Chiloé a un 6,7 \% en Bariloche, en el límite oriental de la distribución del BTSA; Aizen \& Ezcurra 1998), la prevalencia de ornitofilia es relativamente alta si se compara con otras regiones templadas o tropicales del mundo. Por ejemplo, el porcentaje de ornitofilia entre las plantas leñosas es del 3,1 \% (29 especies) en la isla de Barro Colorado, Panamá; 2,9\% (39 especies) en La Selva, Costa Rica; el 2,4 \% (15 especies) en Guanacaste, Costa Rica (Stiles 1985); 7 \% (19 especies) en el Parque Nacional Lambir Hills, Sarawak, Malasia (Momose et al. 1998); 0,8\% (ocho especies) en Montana, Estados Unidos (Lesica 1985); 0,6 \% (1 especie) en el estado de Washington, Estados Unidos (Franklin et al. 1988); y $0 \%$ en el Parque Nacional Kosciusko, Nueva Gales del Sur, Australia (Inouye \& Pyke 1988).

La prevalencia de dispersión por vertebrados que ingieren frutos en el BTSA es también comparativamente alta. Por ejemplo, en la isla de Chiloé el porcentaje de especies de plantas con frutos carnosos es del 65-69\% (Armesto \& Rozzi 1989); este porcentaje es aparentemente mayor que en cualquier otra zona templada del mundo, e incluso mayor que en algunas zonas tropicales. Sólo en selvas tropicales de Australia ( $85 \%$ ), del Neotrópico (70-86\%), y de África occidental (80 $\%$ ) la prevalencia de la endozoocoría en las floras nativas llega a ser mayor (Willson 1991).

Esta alta incidencia de ornitofilia y endozoocoria entre las plantas del BTSA contrasta con la baja riqueza de especies de vertebrados mutualistas. La ornitofauna es pobre comparada con otras regiones (Vuilleumier 1985), lo cual se traduce en pocas especies de aves polinizadoras y dispersoras de semillas. Como mencionamos anteriormente, en el BTSA principalmente una sola especie de picaflor (Sephanoides sephaniodes) es responsable de la polinización de un alto número de especies de plantas ornitófilas. La relación entre el número de especies de aves polinizadoras y el número de plantas ornitófilas está entre las 
más bajas del mundo. En la isla de Chiloé, esta relación es de 0,07 (una especie de polinizador/14 especies de plantas), y sólo es menor en los bosques nublados de Costa Rica $(\sim 0,06,1 / 17-19$ especies), mientras que en otras regiones es considerablemente mayor: 0,21 (4/19 especies) en selvas tropicales en Sarawak, Malasia (Momose et al. 1998); 0,23 (3/13) en bosques secos de Puerto Rico; 0,26 (6/23) en bosques tropicales deciduos de Jalisco, México; 0,44 (4/9) en chaparral y bosque de coníferas en Arizona, Estados Unidos; $1,23(16 / 13)$ en selvas tropicales de Trinidad y Tobago; 1,40 (14/10) en selvas secundarias de Costa Rica; y 1,80 (9/5) en selvas tropicales en la isla de Trinidad (Armesto et al. 1996).

De un modo similar a lo que ocurre con los mutualismos de polinización por aves, existe una baja relación entre animales dispersores de semillas y plantas con síndrome de endozoocoria cuando se compara el BTSA con otros ecosistemas del mundo. Esta relación es de 0,17 (cuatro especies principales de animales/23 especies de plantas) en Chiloé, mientras que en Estados Unidos varía entre 0,54 (22/41) y 1,88 (30/16); en España varía entre $0,71(5 / 7)$ y $1,27(14 / 11)$; en Francia es de 0,31 (20/65); y 0,78 (18/23) en Australia (Armesto et al. 1996).

En Chiloé, el $75 \%$ (45 especies) de las plantas leñosas tienen síndromes de polinización por insectos (Smith-Ramírez \& Armesto 1994) y, a1 menos a nivel de géneros, esta prevalencia aumenta en sentido oeste-este (de 64,9\% de los géneros de leñosas en Chiloé a 76,7 \% en Bariloche; Aizen \& Ezcurra 1998), lo opuesto a lo observado para la ornitofilia. La prevalencia de la entomofilia entre las plantas leñosas es más alta que en la flora boreal holártica, donde alrededor del $60 \%$ de las especies presentan este síndrome (Kevan et al. 1993), pero es más baja que en algunos ecosistemas tropicales, como la estación biológica La Selva en Costa Rica, donde un 82,6 $\%$ (228 especies) de las plantas son polinizadas por insectos (Kress \& Beach 1994).

Los patrones observados en el BTSA en cuanto a la relación entre especies de animales y plantas para los mutualismos de polinización y de dispersión de semillas por aves difieren de los encontrados para los insectos. La relación entre especies de visitantes florales que participan presumiblemente en la polinización y el número de plantas entomófilas en los bosques de Nothofagus dombeyi de Nahuel Huapi es de 4,9 (97 especies de polinizadores/15 especies de plantas; D.P. Vázquez \& D. Simberloff resultados no publicados), mientras que en Chiloé es de 4,6 (76/19 especies); sin embargo, la relación obtenida por Riveros et al. (1991) para Puyehue es considerablemente más baja, 1,6 (50/31 especies), aunque esto probablemente se deba al menor esfuerzo de muestreo en este estudio en comparación con los de SmithRamírez et al. (en prensa, resultados no publicados) y D.P. Vázquez \& D. Simberloff (resultados no publicados) (175 h de observación versus 343 y $452 \mathrm{~h}$, respectivamente). Comparativamente, esta relación es de 13,9 (167/12 especies) para un bosque boreal en Canadá (Barrett \& Helenurm 1987); 4,4 (315/71) para un arbustal xerófilo de Sudáfrica (Struck 1994); 3,0 (79/26) para un pastizal de Inglaterra (Memmott 1999); 2,9 (268/94) para un bosque de coníferas en Colorado, Estados Unidos (Clements \& Long 1923); 2,2 (96/43) para un bosque montano de Nueva Gales del Sur, Australia (Inouye \& Pyke 1988); y de 0,6 (88/ 137) en arbustales de altura en los Andes centrales de Chile (Arroyo et al. 1982). Es decir, dependiendo de si uno considera el estudio de Riveros et al. (1991) o los de Smith-Ramírez et al. (resultados no publicados) y Vázquez \& Simberloff (resultados no publicados) uno puede decir que el BTSA está entre los que tienen una relación más baja o más alta entre el número de insectos polinizadores y el de plantas entomófilas. Esto demuestra lo importante que es la estandarización de los métodos en la comparación de este tipo de datos.

Sin embargo, aún cuando consideremos los estudios de C: Smith-Ramírez (en prensa, resultados no publicados) y de D.P. Vázquez \& D. Simberloff (resultados no publicados) como representativos del BTSA, es necesario tener en cuenta que la gran cantidad de especies de insectos observadas en relación al número de especies de plantas muestreadas nos dice muy poco en cuanto a la estructura funcional del ensamble de plantas e insectos polinizadores. Como hemos mencionado anteriormente, unas pocas especies de insectos son responsables de una gran proporción de las visitas realizadas; sólo unas pocas especies de plantas son visitadas por una gran cantidad de especies de insectos, mientras que la mayoría son visitadas por relativamente pocas especies (Fig. 1 y 2). Los datos presentados gráficamente por Memmott (1999) parecen indicar un patrón similar; lamentablemente, no conocemos otros estudios cuantitativos similares a los de Smith-Ramírez et al. (resultados no publicados), Vázquez \& Simberloff (en prensa, resultados no publicados) y Memmott (1999) que nos permitan realizar comparaciones con otras regiones del mundo. Por otro lado, es claro que la diversidad de insectos polinizadores, aún en términos funcionales, es mucho mayor que la diversidad de vertebrados polinizadores. 


\section{EFECTOS DE PERTURBACIONES ANTRÓPICAS Y CONSERVACIÓN}

El BTSA está experimentando tasas alarmantes de deforestación (Armesto et al. 1998). En Chile, este bioma ha sido casi exterminado en su porción norte, costera y depresión intermedia, esta última ubicada entre la cordillera costera y andina. En este país han desaparecido casi completamente varios tipos forestales (Lara et al. resultados no publicados), siendo reemplazados por ecosistemas agrícolas y forestaciones con especies exóticas. En el lado argentino, si bien la tasa de deforestación no ha sido tan alta, la mayor parte de estos bosques están, en menor o mayor medida, degradados por la presencia de herbívoros introducidos, una alta incidencia de fuego, y tala furtiva. Por ejemplo, el $56 \%$ de la superficie del Parque Nacional Nahuel Huapi está ocupada por ganado vacuno, y un área importante de este parque está invadida por ciervos exóticos (Veblen et al. 1992, Lauría-Sorge \& Romero 1999).

La ruptura de procesos mutualistas como consecuencia de distintos tipos de perturbaciones de origen antrópico es uno de los factores que pueden afectar la biodiversidad (Nabhan \& Fleming 1993, Meffe \& Caroll 1997). La tala de bosques primarios y su reemplazo por vegetación arbustiva conduce muchas veces a formaciones carentes de especies consideradas claves para el BTSA, como es la casi ausencia de Elaenia albiceps en sistemas arbustivos $(2,6 \%$ de abundancia relativa) y en estadios sucesionales tempranos $(7,7 \%$ de abundancia relativa) de los bosques de la isla de Chiloé (Díaz et al. resultados no publicados).

La fragmentación del hábitat remanente que acompaña a la deforestación puede también afectar las relaciones mutualistas de maneras complejas (Aizen \& Feinsinger en prensa). En la isla de Chiloé, por ejemplo, Smith-Ramírez \& Armesto (en prensa) no encontraron diferencias entre el bosque continuo y fragmentos de distinto tamaño en la frecuencia de visitas del colibrí Sephanoides sephaniodes a las flores de Embothrium coccineum. Contrariamente a lo esperado, E. albiceps presentó un aumento en las visitas a las flores de árboles aislados de E. coccineum y de árboles en fragmentos pequeños. Si bien estos resultados no sugieren la existencia de un efecto negativo de la fragmentación sobre la polinización de esta planta, Willson et al. (2001) conside-

${ }^{3}$ HENRIQUEZ C, J SIMONETTI \& R BUSTAMANTE (2001) Éxito reproductivo de Lapageria rosea en bosques fragmentados. Primera Reunión Binacional de Ecología Chile-Argentina, San Carlos de Bariloche, Libro resúmenes: 274 . ran que los mutualismos ave-planta en los paisajes fragmentados podrían estar en riesgo si el éxito reproductivo de los animales mutualistas es afectado. Aunque existe un cierto grado de generalismo en $S$. sephaniodes, E. albiceps y Turdus falcklandii en cuanto a la utilización de fragmentos de un amplio rango de tamaños (Willson et al. 1994), su éxito de nidificación en fragmentos pequeños, borde de fragmentos y corredores es aparentemente bajo (Willson et al. 2001). Por otra parte, Cornelius et al. (1999) postulan que existe un cierto grado de resistencia a la perturbación por fragmentación en estas especies. En fragmentos de BTSA aislados desde fines del Terciario en el norte chico de Chile $\left(30^{\circ}\right.$ $40^{\prime} \mathrm{S}$ ), ellos encontraron que estos mutualistas, además del furnárido Aphrastura spinicauda, eran las especies de aves más abundantes. Sin embargo, estos autores consideran a los mutualistas frugívoros menos frecuentes (e.g., Curaeus curaeus y Phytotoma rara) como especies perdidas o extintas en estos fragmentos no conectados con el bosque templado continuo de más al sur.

La pérdida de bosques primarios y su reemplazo por otros usos y por bosques secundarios, puede afectar la permanencia de muchas especies vegetales y presumiblemente de sus animales mutualistas. Por ejemplo, las poblaciones de las gesneriáceas ornitófilas Asteranthera ovata y Sarmienta repens parecen ser muy propensas al desecamiento. A estas especies sólo se las encuentra en hábitats de bosque primario, donde suelen ser muy abundantes, y en algunos bosques secundarios donde la alteración no ha sido muy alta. Lapageria rosea, Crinodendron hookerianum, Philesia magellanica y Desfontainia spinosa son otras especies polinizadas por picaflores y con frutos carnosos que raramente se encuentran en bosques secundarios o altamente degradados (C. Smith-Ramírez observación personal). En el caso del copihue, Lapageria rosea, Henríquez et al. (2001) ${ }^{3}$ encontraron que la fragmentación de hábitat afecta el éxito reproductivo de esta especie a través de una disminución en la producción de frutos y un aumento en la depredación de semillas.

El efecto de las alteraciones de origen antrópico no está, sin embargo, restringido a las plantas polinizadas por aves. Recientemente D.P. Vázquez (resultados no publicados) encontró efectos indirectos de los herbívoros introducidos sobre la reproducción de Alstroemeria aurea. Esta especie es altamente susceptible al pisoteo, y en consecuencia disminuye en densidad en bosques con ungulados introducidos. Esta menor densidad se traduce en una menor frecuencia de visitas de los polinizadores, resultando en un menor depósito 
de polen en los estigmas y una mayor contaminación con polen de otras especies; esto a su vez resulta en una menor producción de frutos y semillas. Aunque A. aurea depende para su éxito reproductivo de un número relativamente bajo de especies de polinizadores (Aizen 1997, 2001, D.P. Vázquez resultados no publicados), esta planta representa, sin embargo, un recurso importante para muchas especies de polinizadores (Fig. 1A), siendo prácticamente la única especie en flor durante una buena parte del verano en los bosques de Nothofagus dombeyi. De este modo, los herbívoros introducidos podrían estar afectando indirectamente a un número relativamente alto de especies de insectos que dependen del amancay.

La fragmentación o la degradación del bosque pueden también tener efectos importantes sobre las poblaciones de algunos mamíferos mutualistas. En el caso de Dromiciops gliroides, RodríguezCabal et al. (2001) estimaron abundancias de hasta 40-70 ind ha ${ }^{-1}$ en parcelas con bosque maduro y continuo de Nothofagus dombeyi en el bosque de Llao-Llao. Sin embargo, la abundancia de este animal decreció prácticamente a cero en parcelas vecinas ocupadas por bosque fragmentado y degradado. Estos autores observaron una disrupción del mutualismo entre este marsupial y la hemiparásita Tristerix corymbosus en el bosque fragmentado, registrando una baja tasa de remoción de frutos y una ausencia casi total de semillas dispersadas y nuevas plántulas establecidas.

En general, todavía es poco lo que se sabe sobre el efecto de la fragmentación sobre los polinizadores invertebrados tanto en el BTSA como en otros biomas (Aizen \& Feinsinger en prensa). En particular, poco conocemos sobre cuáles especies de polinizadores se pierden, cómo cambian los ensambles y cómo estos cambios, de existir, afectan el éxito reproductivo de las plantas. En las cercanías de Puerto Varas, Riveros et al. (manuscrito) encontraron que las flores del ulmo (Eucryphia cordifolia) eran visitadas por un ensamble muy reducido de polinizadores compuesto por sólo cuatro especies y dominado por Apis mellifera con casi un $90 \%$ del total de las visitas. En contraste, en bosques secundarios conectados con bosques primarios de Chiloé, C. Smith-Ramírez et al. (resultados no publicados) registraron 54 especies de polinizadores en las flores de este árbol con un aporte de Apis mellifera de sólo el $37 \%$ del total de visitas.

${ }^{4}$ RODRÍGUEZ-CABAL MA, MA AIZEN \& AJ NOVARO (2001) Dispersión de las semillas del quintral (Tristerix corymbosus) por el monito del monte (Dromiciops australis): efectos de la perturbación antrópica del hábitat. Primera Reunión Binacional de Ecología, San Carlos de Bariloche, Libro resúmenes: 290.
Recientemente, dos especies de himenópteros de origen europeo fueron introducidos en el BTSA, el abejorro Bombus ruderatus y la chaqueta amarilla, Vespula germanica. Bombus ruderatus fue liberado intencionalmente en Chile (Arretz \& Mcfarlane 1986), dispersándose posteriormente a la Argentina (Roig-Alsina \& Aizen 1996). Aunque $B$. ruderatus es un actor activo en la polinización de algunas de las especies polinizadas por el nativo Bombus dahlbomii, el abejorro exótico no participa en la polinización de otras plantas visitadas por este último. Por ejemplo, en Nahuel Huapi $B$. ruderatus frecuentó las flores de Alstroemeria aurea durante los veranos del 2000 y del 2001 con un $14 \%$ de las visitas totales (D.P. Vázquez \& D. Simberloff resultados no publicados), pero virtualmente no intervino en la polinización de Berberis darwinii, Mutisia decurrens, Vicia nigricans y la éxotica Digitalis purpurea, todas ellas polinizadas principalmente por B. dahlbomii. Esto probablemente se deba, al menos en parte, a la marcada diferencia en la fenología de ambas especies de abejorros: las reinas de B. dahlbomii se encuentran activas desde el inicio de la temporada de floración, en la primavera temprana (septiembre-octubre), mientras que $B$. ruderatus recién aparece hacia el final de la primavera (Fig. 3). El reemplazo de $B$. dahlbomii por $B$. ruderatus podría afectar a las especies que son visitadas sólo por el abejorro nativo. De hecho, la abundancia de $B$. ruderatus ha aumentado al este de los Andes desde que esta especie fuera observada por primera vez en Argentina en 1993 (Roig-Alsina \& Aizen 1996). Por ejemplo, en una población de Alstroemeria aurea del cerro Chall-huaco, Aizen (2001) registró en los veranos de 1994 y 1995 una frecuencia de visitas extremedamante baja $(<1 \%$ del total) por parte de B. ruderatus. En 1996, este autor observó que $B$. ruderatus realizó casi el $5 \%$ del total de visitas recibidas por $A$. aurea en la población de Chall-huaco, mientras que en 1998 la frecuencia aumentó a casi el 50 \% (M.A. Aizen resultados no publicados). Sin embargo, de los ocho sitios estudiados por Vázquez \& Simberloff (en prensa) en los veranos de 2000 y 2001, en cuatro se observó un aumento de la frecuencia de $B$. ruderatus a las flores de $A$. aurea de un año a otro mientras que en tres sitios se observó una disminución (en el octavo sitio no se registraron visitas de ninguno de los dos abejorros en 2001). Esta tendencia podría estar indicando una estabilización en la abundancia de este abejorro exótico a nivel regional luego de la explosión demográfica registrada durante los primeros 5 años desde su arribo a la región de Nahuel Huapi (Roig-Alsina \& Aizen 1996). 
Vespula germanica fue introducida en Chile en 1974 (Peña et al. 1975). A principios de los 80 ya estaba en el lado oriental de los Andes en la provincia del Neuquén, Argentina (Willink 1980), y es ahora uno de los insectos depredadores dominantes en el norte del BTSA y en el oeste de la estepa patagónica (Farji-Brenner \& Corley 1998). Esta especie ha invadido también zonas de los Estados Unidos, Australia, Nueva Zelandia, la isla Ascención y Sudáfrica (Clout \& Lowe 2000). En general $V$. germanica es altamente generalista, alimentándose de nectar, miel de abejas domésticas, otros artrópodos, vertebrados muertos y restos de comida humana. En el BTSA, por ejemplo, $V$. germanica se alimenta de artrópodos de al menos nueve órdenes distintos, entre los que se encuentran polinizadores como abejas y sírfidos (Sackmann et al. 2000). Aunque aún no existe información suficiente para evaluar el impacto de $V$. germanica, es posible que tenga efectos importantes en la fauna de invertebrados del BTSA. Es posible, además que estos efectos se transmitan a través de la red de interacciones, sin poder descartarse un efecto sobre la polinización.

Las perturbaciones de origen antrópico favorecerían tanto la invasión de plantas como de visitantes florales exóticos (Aizen \& Feinsinger en prensa). Recientemente, Morales \& Aizen (en prensa) registraron en la región Nahuel Huapi un incremento de casi el $300 \%$ en la frecuencia de visitas por polinizadores exóticos (Bombus ruderatus y Apis mellifera) en sitios altamente perturbados, en comparación con sitios con un bajo grado de perturbación antrópica. Además, estos autores encontraron que las flores de plantas exóticas recibieron-tanto en sitios altamente como poco perturbados-en promedio 2,5 veces más visitas que las flores de plantas nativas, incluso por especies de polinizadores nativos. Esto plantearía la posibilidad de un escenario de competencia entre plantas nativas y exóticas a través de sus polinizadores (Feinsinger 1987b).

Hoy en día existe un conflicto entre la conservación de la biodiversidad en sus distintos niveles de organización vs. la conservación del funcionamiento de los ecosistemas (Kunin \& Lawton 1996). En el caso del BTSA, la gran mayoría de los visitantes florales invertebrados parecerían no tener una gran importancia desde el punto de vista funcional, ya que su contribución a la polinización de las plantas que visitan es aparentemente muy baja (ya sea en términos de la frecuencia de visitas o, en algunos casos, en cuanto a la cantidad de polen que llevan). Sin embargo, tal como ocurre en otros grupos taxonómicos, la fauna de insectos del BTSA se caracteriza por un alto grado de endemismo. Por ejemplo, esta re- gión tiene una fauna de abejas extremadamente singular, en la que están ausentes muchos de los grupos neotropicales como las Meliponinae y las Euglosinae, pero presentes varios grupos endémicos y probablemente relictuales, tales como las Xeromelissinae, Diphaglossa, Cadeguala, Corynura, Neofidelia, Manuelia, y Eucerinoda (Michener 1979). Lo mismo sucede con los dípteros, por ejemplo, de la familia Nemestrinidae. Un énfasis demasiado fuerte en la conservación de los aspectos funcionales de las interacciones entre plantas y animales mutualistas podría dar por tierra con muchos de estos grupos de baja importancia funcional pero que contribuyen enormemente a la diversidad filogenética.

\section{AGRADECIMIENTOS}

Nuestro agradecimiento a Juan Armesto, Vanina Chalcoff, Ivan Díaz, Carolina Henríquez, Carolina Morales y Mariano Rodríguez-Cabal por perminitirnos citar sus trabajos aún no publicados. También agradecemos a Anna Traveset por su insistencia y apoyo para que realicemos esta contribución. A Guillermo Amico, Juan Corley, Carolina Morales, y Adriana Ruggiero por sus comentarios sobre una versión preliminar de este manuscrito. Distintos aspectos de este trabajo fueron financiados por la Fundación Antorchas (proyecto A-13740/1-14), FONCYT (PICT 0107320), Wildlife Conservation Society, University of Tennessee, National Science Foundation, Turner Foundation, Foundation for Deep Ecology, Centro-Núcleo Milenio para Estudios Avanzados en Ecología e Investigación (P99103FICM) y una Cátedra Presidencial en Ciencias, otorgada al Dr. Juan Armesto. El primer autor es miembro de la Carrera de Investigador del CONICET.

\section{LITERATURA CITADA}

AIZEN MA (1997) Influence of local floral density and flower-phase sex ratio on pollen receipt and seed output: empirical and experimental results in dichogamous Alstroemeria aurea (Alstroemeriaceae). Oecologia 111: 404-412.

AIZEN MA (2001) Flower sex ratio, pollinator abundance, and the seasonal pollination dynamics of a protandrous plant. Ecology 82: 127-144.

AIZEN MA \& C EZCURRA (1998) High incidence of plant-animal mutualisms in the woody flora of the temperate forest of southern South America: biogeographical origin and present ecological significance. Ecología Austral 8: 217-236. 
AIZEN MA \& P FEINSINGER (en prensa) Bees not to be? responses of insect pollinator faunas and flower pollination to habitat fragmentation. En: Bradshaw GA, P Marquet \& HA Mooney (eds) How landscapes change: human disturbance and ecosystem disruptions in the Americas. Springer-Verlag, New York, New York.

AMICO G (2000) Dispersión del quintral (Tristerix corymbosus) por el monito del monte (Dromiciops australis). Tesis de Licenciatura, Centro Regional Bariloche, Universidad Nacional del Comahue, Bariloche, Argentina. 59 pp.

AMICO G \& MA AIZEN (2000) Mistletoe seed dispersal by a marsupial. Nature 408: 929-930.

ARAYA MB \& GH MILLIE (1986) Guía de campo de las aves de Chile. Editorial Universitaria, Santiago, Chile. $406 \mathrm{pp}$.

ARMESTO JJ \& R ROZZI (1989) Seed dispersal syndromes in the rain forest of Chiloé: evidence for the importance of biotic dispersal in a temperate rain forest. Journal of Biogeography 16: 219-226.

ARMESTO JJ, R ROZZI, P MIRANDA \& C SABAG (1987) Plant/frugivore interactions in South American temperate forests. Revista Chilena de Historia Natural 60: 321-336.

ARMESTO JJ, C SMITH-RAMÍREZ \& C SABAG (1996) The importance of plant-bird mutualisms in the temperate rainforest of southern South America. En: Lawford RG, PB Alaback \& E Fuentes (eds) Highlatitude rainforests and associated ecosystems of the west coast of the Americas: 248-265. Springer-Verlag, New York, New York.

ARMESTO JJ, R ROZZI, C SMITH-RAMÍREZ \& MTK ARROYO (1998) Conservation targets in South American temperate forests. Science 282: 1271- 1272.

ARRETZ PV \& RP MACFARLANE (1986) The introduction of Bombus ruderatus to Chile for red clover pollination. Bee World 67: 15-22.

ARROYO MTK \& A PEÑALOZA (1990) Genetic selfcompatibility in a South American species of Ourisia (Scrophulariaceae). New Zealand Journal of Botany 18: 467-470.

ARROYO MTK \& AE HOFFMAN (1997) Temperate rain forest of Chile. En: Davis SD, O Herrera-MacBryde, J Villa-Lobos \& AC Hamilton (eds) Centres of plant diversity, Volume 3: The Americas: 542-548. World Wildlife Fund y International Union for Conservation of Nature, New York.

ARROYO MTK, RB PRIMACK \& JJ ARMESTO (1982) Community studies in pollination ecology in the high temperate Andes of Central Chile. I. Pollination mechanisms and altitudinal variation. American Journal of Botany 69: 82-97.

ARROYO MTK, L CAVIERES, A PEÑAlOZA, M RIVEROS \& AM FAGGI (1996) Relaciones fitogeográficas y patrones regionales de riqueza de especies en la flora del bosque lluvioso templado de Sudamérica. En: Armesto JJ, C Villagrán \& MTK Arroyo (eds) Ecología de los bosques nativos de Chile: 71-99. Editorial Universitaria, Santiago, Chile.
BAKER HG \& I BAKER (1983) Floral nectar constituents in relation to pollinator type. En: Jones CE \& RJ Little (eds) Handbook of experimental pollination biology: 117-141. Van Nostrand Reinhold Company, New York, New York.

BARRETT SCH \& K HELENURM (1987) The reproductive biology of boreal forest herbs. I. Breeding systems and pollination. Canadian Journal of Botany 65: 20362046.

BAWA KS (1990) Plant-pollinator interactions in tropical rain forests. Annual Review of Ecology and Systematics 21: 399-422.

BOND WJ (1994) Do mutualisms matter? assessing the impact of pollinator and disperser disruption on plant extinction. Philosophical Transactions of the Royal Society of London B Biological Sciences 344: 83-90.

CABRERA AL \& A WILLINK (1980) Biogeografía de América Latina. Organización de Estados Americanos, Washington, District of Columbia. vi +120 pp.

CLEMENTS RE \& FL LONG (1923) Experimental pollination: an outline of the ecology of flowers and insects. Carnegie Institute of Washington, Washington, District of Columbia.

CLOUT MN \& SJ LOWE (2000) Invasive species and environmental changes in New Zealand. En: Mooney HA \& RJ Hobbs (eds) Invasive species in a changing world: 369-383. Island Press, Washington, District of Columbia.

CORNELIUS C, H COFRÉ \& PA MARQUET (1999) Effects of habitat fragmentation on bird species in a relict temperate forest in semiarid Chile. Conservation Biology 14: 534-543.

CORREA A, JJ ARMESTO, RP SCHLATTER, R ROZZI \& JC TORRES-MURA (1990) La dieta del chucao (Scelorchilus rubecula), un Passeriforme terrícola endémico del bosque templado húmedo de Sudamérica austral. Revista Chilena de Historia Natural 63: 197 202.

DINERSTEIN E, DM OLSON, DJ GRAHAM, AL WEBSTER, SA PRIMM, MP BOOKINDER \& G LEDEC (1995) Una evaluación del estado de conservación de las ecorregiones terrestres de América Latina y el Caribe. Banco Mundial/World Wildlife Fund.

DUELLMAN WE (1999) Distribution patterns of amphibians in South America. En: Duellman WE (ed) Patterns of distribution of amphibians: a global perspective: 255-328. Johns Hopkins University Press, Baltimore, Maryland.

FARJI-BRENNER A \& JC CORLEY (1998) Successful invasions of hymenopteran insects into NW Patagonia. Ecología Austral 8: 273-249.

FEINSINGER P (1987a) Approaches to nectarivore-plant interactions in the New World. Revista Chilena de Historia Natural 60: 285-319.

FEINSINGER P (1987b) Effects of plant species on each other's pollination: is community structure influenced? Trends in Ecology and Evolution 2: 123126.

FLEMING TH (1991) Fruiting plant-frugivore mutualism: the evolutionary theater and the ecological play. En: Price PW, GW Fernandes, TM Lewinsohn \& WW Benson (eds) Plant-animal interactions: evolutionary ecology in tropical and temperate regions: 119-144. John Wiley \& Sons, New York, New York. 
FOLGARAIT PJ, A MONJEAU \& M KITTLEIN (1998) Solving the enigma of granivory rates in Patagonia and throughout other deserts of the world: is thermal range the explanation? Ecología Austral 8: 251-264.

FRAGA R, AE RUFFINI \& D GRIGERA (1997) Interacciones entre el picaflor rubí Sephanoides sephaniodes y plantas del bosque subantártico en el Parque Nacional Nahuel Huapi, Argentina. El Hornero 14: 224-234.

FRANKLIN JF, WH MOIR, MA HEMSTROM, A MILES, SE GREENE \& BG SMITH (1988) Forest communities of Mount Rainier National Park. United States Department of Agriculture, National Park Service, Atlanta, Georgia. 194 pp.

GARCÍA JR (1982) Comunidad avifaunística del delta del río Golgol, una necesidad de conservación. Tesis de Licenciatura, Instituto de Zoología, Universidad Austral de Chile, Valdivia, Chile. 126 pp.

GRIGERA D, CA UBEDA \& S CALI (1994) Caracterización ecológica de la asamblea de tretápodos del Parque y Reserva Nacional Nahuel Huapi, Argentina. Revista Chilena de Historia Natural 67: 273-298.

HERNÁNDEZ JF (1995) Efecto de los árboles percha sobre los patrones de lluvia de semillas en el establecimiento de plántulas: consecuencias para la sucesión secundaria del bosque de Chiloé. Tesis de Doctorado en Ciencias, Facultad de Ciencias, Universidad de Chile, Santiago, Chile. 119 pp.

HEYWOOD VH (1995) Global biodiversity assessment. Cambridge University Press, Cambridge, United Kingdom. 1140 pp.

HOFFMAN AJ, ER FUENTES, I CORTES, F LIBERONA \& V COSTA (1986) Tristerix tetrandrus (Loranthaceae) and its host-plants in the Chilean matorral: patterns and mechanisms. Oecologia 69: 202-206.

INOUYE DW \& GH PYKE (1988) Pollination biology in the Snowy Mountains of Australia: comparisons with montane Colorado, USA. Australian Journal of Ecology 13: 191-210.

JAKSIC F (1997) Ecología de los vertebrados de Chile. Ediciones Universidad Católica de Chile, Santiago, Chile. 262 pp.

KEARNS CA \& DW INOUYE (1997) Pollinators, flowering plants, and conservation biology. Bioscience 47: 297-306.

KELT DA \& DR MARTÍNEZ (1989) Notes on distribution and ecology of two marsupials endemic to the Valdivian forests of southern South America. Journal of Mammalogy 70: 220-224.

KEVAN PG, EA TIKHMENEV \& M USUI (1993) Insects and plants in the pollination ecology of the boreal zone. Ecological Research 8: 247-267.

KRESS WJ \& JH BEACH (1994) Flowering plant reproductive systems. En: McDade LA, KS Bawa, HA Hespenheide \& GS Hartshorn (eds) La Selva: ecology and natural history of a neotropical rain forest: 161-182. University of Chicago Press, Chicago, Illinois.

KUIJT J (1988) Revision of Tristerix (Loranthaceae). Systematic Botany Monographs 19: 1-61.
KUNIN WE \& JH LAWTON (1996) Does biodiversity matter? evaluating the case for conserving species. En: Gaston KJ (eds) Biodiversity: a biology of numbers and difference: 283-308. Blackwell Scientific Publications, Oxford, United Kingdom.

LAURIA-SORGE RM \& CA ROMERO (1999) La Ganadería doméstica de los pobladores con Permiso de Ocupación y Pastaje (P.P.O.P) en tierras fiscales del Parque Nacional Nahuel Huapi. Administración de Parques Nacionales-Intendencia Parque Nacional Nahuel Huapi, Bariloche, Argentina. 35 pp.

LESICA P (1985) Checklist of the vascular plants of Glacier National Park, Montana, USA. Monograph No. 4, Montana Academy of Sciences, Missoula, Montana. $55 \mathrm{pp}$.

MARONE L, J LÓPEZ DE CASENAVE \& VR CUETO (2000) Granivory in southern south American deserts: conceptual issues and current evidence. Bioscience 50: 123-132.

MARTÍNEZ DEL RÍO C, HG BAKER \& I BAKER (1992) Ecological and evolutionary implications of digestive processes: bird preferences and the sugar constituents of floral nectar and fruit pulp. Experientia 48: 544551.

MEFFE G \& C CARROLL (1997) Principles of conservation biology. Second edition. Sinauer Associates, Inc., Sunderland, Massachusetts. $\mathrm{xx}+$ $600 \mathrm{pp}$.

MEMMOTT J (1999) The structure of a plant-pollinator food web. Ecology Letters 2: 276-280.

MESERVE PL, BK LANG \& BD PATTERSON (1988) Trophic relationships of small mammals in a Chilean temperate rainforest. Journal of Mammalogy 69: 721730.

MICHENER CD (1979) Biogeography of bees. Annals of the Missouri Botanical Garden 66: 277-347.

MOMOSE K, T YUMOTO, T NAGAMITSU, M KATO, H NAGAMASU, S SAKAI, RD HARRISON, T ITIOKA, AA HAMID \& T INOUE (1998) Pollination biology in a lowland dipterocarp forest in Sarawak, Malaysia. I. Characteristics of the plant-pollinator community. American Journal of Botany 85: 1477-1501.

MORALES CL \& MA AIZEN (en prensa) Does invasion of exotic plants promote invasion of exotic flower visitors? A case study from the temperate forest of the southern Andes. Biological Invasions.

MORRONE JJ \& S ROIG-JUÑENT (1995) The diversity of Patagonian weevils. Ediciones LOLA, Buenos Aires, Argentina. 189 pp.

NABHAN GP \& T FLEMING (1993) The conservation of mutualisms. Conservation Biology 7: 457-459.

PEÑA LE, R PÉREZ ARCE \& L CARTAGENA (1975) La presencia de Vespula maculifrons (Buysson) (Hymenoptera:Vespidae) en Chile. Revista Chilena de Entomología 9: 167-168.

RAU JR, DR MARTÍNEZ, JR LOW \& MS TILLERIA (1995) Depredación de zorros chillas (Pseudalopex griseus) sobre micromamíferos cursoriales, escansoriales, y arborícolas en un área silvestre protegida del sur de Chile. Revista Chilena de Historia Natural 68: 333-340.

REID N (1991) Coevolution of mistletoes and frugivorous birds. Australian Journal of Ecology 16: 457-469. 
RIVEROS M (1991) Aspectos sobre la biología reproductiva en dos comunidades del sur de Chile, $40^{\circ} \mathrm{S}$. Tesis de Doctorado en Ciencias, Facultad de Ciencias, Universidad de Chile, Santiago, Chile. xxxi $+301 \mathrm{pp}$.

RIVEROS M, AM HUMAÑA \& D LANFRANCO (1991) Actividad de los polinizadores en el Parque Nacional Puyehue, X Región, Chile. Medio Ambiente (Chile) 11: 5-12.

ROIG-ALSINA (1999) Revisión de las abejas colectoras de aceites del género Chalepogenus Holmberg (Hymenoptera, Apidae, Tapinotaspidini). Revista del Museo Argentino de Ciencias Naturales 1: 67-101.

ROIG-ALSINA A \& MA AIZEN (1996) Bombus ruderatus Fabricius, un nuevo Bombus para la Argentina (Hymenoptera: Apidae). Physis (Argentina) Sección C 51: 49-50.

ROZZI R, D MARTÍNEZ, MF WILLSON \& C SABAG (1996) Avifauna de los Bosques Templados de Sudamérica. En: Armesto JJ, C Villagrán \& MTK Arroyo (eds) Ecología de los bosques nativos de Chile: 135-152. Editorial Universitaria, Santiago, Chile.

RUFFINI AE (1992) Interacciones entre Sephanoides sephaniodes (Molina) y las plantas que poliniza en el bosque de Nothofagus. Tesis de Licenciatura, Centro Regional Bariloche, Universidad Nacional del Comahue, Bariloche, Argentina. 81 pp.

SABAG C (1993) El rol de las aves en la dispersión de semillas en un bosque templado secundario de Chiloé $\left(42^{\circ} \mathrm{S}\right)$. Tesis de Maestría, Facultad de Ciencias, Universidad de Chile, Santiago, Chile. 79 pp.

SACKMANN P, P D'ADAMO, M RABINOVICH \& JC CORLEY (2000) Arthropod prey foraged by the German wasp (Vespula germanica) in NW Patagonia, Argentina. New Zealand Entomologist 23: 55-59.

SMITH-RAMÍREZ C (1993) Los picaflores y su recurso floral en el bosque templado de la isla de Chiloé, Chile. Revista Chilena de Historia Natural 66: 65-73.

SMITH-RAMÍREZ C \& JJ ARMESTO (1994) Flowering and fruiting patterns in the temperate rainforest of Chiloé, Chile-ecologies and climatic contraints. Journal of Ecology 82: 353-365.

SMITH-RAMÍREZ C \& JJ ARMESTO (1998) Nectarivoría y polinización por aves en Embothrium coccineum (Proteaceae) en el bosque templado del sur de Chile. Revista Chilena de Historia Natural 71: 51-63.

SMITH-RAMÍREZ C \& JJ ARMESTO (en prensa) Foraging behaviour of nectar-feeding birds visiting trees in edges of forest fragments and fields in southern Chile. Austral Ecology.

SPOTORNO AE, JC MARÍN, M YEVENES, LI WALKER, R FERNÁNDEZ, S BERRÍOS, J PINCHEIRA \& E PALMA (1997) Chromosome divergences among American marsupials and the Australian affinities of the American Dromiciops. Journal of Mammal Evolution 4: 259-269.

STATTERSFIELD AJ, MJ CROSBY, AJ LONG \& DC WEGE (1998) Endemic bird areas of the world: Priorities for biodiversity conservation. BirdLife International, Cambridge, United Kingdom. 846 pp.
STILES FG (1985) Conservation of forest birds in Costa Rica: problems and perspectives. En: Diamond AW \& TE Lovejoy (eds) Conservation of tropical forest birds: 141-168. International Council for Bird Preservation, Cambridge, United Kingdom.

STRUCK M (1994) Flowers and their insect visitors in the arid winter rainfall region of southern Africa: observations on permanent plots. Composition of the anthophilous insect fauna. Journal of Arid Environments 28: 45-50.

TAMBUSSI C \& J NORIEGA (1996) Summary of the avian fossil record from southern South America. Muenchner Geowissenschaftliche Abhandlungen 30: 245-264.

TRAVESET A (1998) Effect of seed passage through vertebrate frugivores' guts on germination: a review. Perspectives in Plant Ecology, Evolution and Sytematics 1/2: 151-190.

TRAVESET A, MF WILLSON \& C SABAG (1998) Effect of nectar-robbing birds on fruit set of Fuchsia magellanica in Tierra del Fuego: a disrupted mutualism. Functional Ecology 12: 459-464.

VÁZQUEZ DP \& D SIMBEROFF (en prensa) Ecological specialization and susceptibility to disturbance: conjectures and regulations. American Naturalist.

VEBLEN TT, M MERMOZ, C MARTÍN \& T KITZBERGER (1992) Ecological impacts of introduced animals in Nahuel Huapi National Park, Argentina. Conservation Biology 6: 71-83.

VILLAGRÁN C, JJ ARMESTO \& R LEIVA (1986) Recolonización postglacial de Chiloé insular: evidencias basadas en la distribución geográfica y los modos de dispersión de la flora. Revista Chilena de Historia Natural 59: 19-39.

VUILLEUMIER F (1985) Forest birds of Patagonia: ecology, geography, speciation, endemism, and faunal history. Ornithological Monographs 36: 255-302.

WILLINK A (1980) Sobre la presencia de Vespula germanica (Fabricius) en la Argentina (Hymenoptera: Vespidae). Neotropica 26: 205-206.

WILLSON MF (1991) Dispersal of seeds by frugivorous animals in temperate forests. Revista Chilena de Historia Natural 64: 537-554.

WILLSON MF, AK IRVINE \& NG WALSH (1989) Vertebrate dispersal syndromes in some Australian and New Zealand plant communities, with geographic comparisons. Biotropica 21: 133-147.

WILLSON MF, T DE SANTO, C SABAG \& JJ ARMESTO (1994) Avian communities in fragmented Southtemperate rainforest in Chile. Conservation Biology 8: 508-520.

WILLSON MF, C SMITH-RAMÍREZ, C SABAG \& JH HERNÁNDEZ (1996a) Mutualismos entre plantas y animales en bosques templados de Chile. En: Armesto JJ, C Villagrán \& MTK Arroyo (eds) Ecología de los bosques nativos de Chile: 251-264. Editorial Universitaria, Santiago, Chile.

WILLSON MF, C SABAG, J FIGUEROA, JJ ARMESTO \& M CAVIEDES (1996b) Seed dispersal by lizards in Chilean rainforest. Revista Chilena de Historia Natural 69: 339-342. 
WILLSON MF, C SABAG, J FIGUEROA \& JJ ARMESTO (1996c) Frugivory and seed dispersal of Podocarpus nubígena in Chiloé, Chile. Revista Chilena de Historia Natural 69: 343-349.
WILLSON MF, JL MORRISON, KE SIEVING, T DE SANTO, L SANTISTEB AN \& I DÍAZ (2001) Patterns of predation risk and survival of bird nests in a Chilean agricultural landscape. Conservation Biology 15: 447-456.

Editor Asociado: R. Medel

Recibido el 30 de abril de 2001; aceptado el 8 de enero de 2002 